\title{
Twelve-month Outcomes of a Cluster-randomized Trial of Home-based Postpartum Contraceptive Delivery in Southwest Trifinio, Guatemala
}

Margo Harrison ( $\sim$ margo.harrison@cuanschutz.edu )

University of Colorado Hospital https://orcid.org/0000-0003-2940-2241

\section{Saskia Bunge-Montes}

FSIG

\section{Claudia Rivera}

FSIG

\section{Andrea Jimenez-Zambrano}

University of Colorado Hospital Aurora: University of Colorado - Anschutz Medical Campus

\section{Gretchen Heinrichs}

Denver Health and Hospital Authority

\section{Antonio Bolanos}

FSIG

\section{Edwin Asturias}

University of Colorado Anschutz Medical Campus: University of Colorado - Anschutz Medical Campus

\section{Steven Berman}

University of Colorado Anschutz Medical Campus: University of Colorado - Anschutz Medical Campus Jeanelle Sheeder

University of Colorado Anschutz Medical Campus: University of Colorado - Anschutz Medical Campus

\section{Research}

Keywords: postpartum contraception, implant, Guatemala

Posted Date: December 23rd, 2020

DOI: https://doi.org/10.21203/rs.3.rs-132684/v1

License: (c) (i) This work is licensed under a Creative Commons Attribution 4.0 International License.

Read Full License 


\section{Abstract}

Design: We executed a cluster-randomized parallel arm pragmatic trial to observe the association of home-based postpartum contraceptive provision, including the contraceptive implant, with three and 12month contraceptive utilization, satisfaction, and pregnancy rates.

Methods: Eight clusters were randomized to receive either the home-based contraceptive delivery (condoms, pills, injection, implant) during the routine 40-day postpartum visit in addition to routine care, or routine care alone, which included comprehensive contraceptive counseling throughout antepartum care.

Results: 208 women were enrolled in the study, 108 in the intervention clusters and 100 in control clusters. 94 (87.0\%) women in the intervention group and 91 (91\%) of women in control clusters were evaluated 12 months post-enrollment. Likelihood of using contraception at that time was borderline increased in intervention clusters (RR $1.1[1.0,1.3], p=0.05)$ with an increased likelihood of long-acting contraceptive use (the implant; RR 1.6 [1.3,1.9], $p<0.001$ ). Pregnancy rates were also borderline reduced in the intervention clusters (RR 1.0 [1.0,1.1], $p=0.07$ ). There was no difference in satisfaction of women with contraceptive use between arms with about $95 \%$ of women very satisfied or a little satisfied in each arm. Continuation rates at twelve months of contraceptives in the intervention group were $0.0 \%$ for condom users, $80.0 \%$ for contraceptive pill users, $57 \%$ for injectable users, and $83 \%$ for implant users. Most women who discontinued their initial method chose a more long-term or permanent method. There was a trend toward a significant association with reduced short interval pregnancy.

Conclusion: Our study had a borderline increase in overall use of contraception by 12 months, did have an increased likelihood of long-acting contraceptive use of the implant by 12 months, and resulted in a trend toward reduced short interval pregnancy in the intervention clusters as compared to control clusters.

Registration: clinicaltrials.gov, NCT04005391;

Retrospectively Registered 7/2/2019, https://clinicaltrials.gov/ct2/show/NCT04005391

Protocol: https://doi.org/10.1186/s13063-019-3735-3

\section{Plain English Summary:}

In a rural area in the Southwest of the country, there is a region where a large migrant worker community resides. The community is served by community-based nursing programs that provide maternal and child health in the home setting. To respond to access barriers to the use of long-acting contraceptives, we conducted a cluster-randomized trial where the intervention group received a home-based postpartum contraceptive intervention. To execute the intervention the nurses brought contraceptives (including the long-acting implant) to women's forty-day postpartum visit, and the control group received standard postpartum care, which included education about postpartum contraception, but methods were not 
provided. Our primary outcome showed that our intervention increased contraceptive utilization at three months in the intervention group compared to the control, which has been published. When we surveyed the women at twelve months post-enrollment, we found that the rate of contraceptive uptake in the intervention communities was almost higher overall than in the control communities, and that the rate of implant use was significantly higher. There was also a trend toward reduced short interval pregnancy.

\section{Introduction:}

We conducted a cluster-randomized, pragmatic, parallel arm trial whereby study nurses brought contraceptives (condoms, pills, injection, implant) to the homes of postpartum women in intervention clusters.. ${ }^{1}$ The objective of the study was to observe whether providing contraceptives at a 40 day postpartum home visit was associated with an increase in implant uptake by three months postpartum. ${ }^{1}$ Our results found that our intervention was successful and increased overall contraceptive uptake (RR 1.3 $[1.1,1.5], p<0.001)$ as well as implant uptake by three months (RR 1.3 [1.2,1.4]) with high satisfaction and no adverse outcomes. ${ }^{2}$ The objective of this additional analysis is to present the 12-month continuation, satisfaction, and pregnancy rates of the study, which represent secondary outcomes of the trial. ${ }^{1}$ We hypothesized based on prior literature that our trial would have high rates of contraceptive continuation and satisfaction and likely reduce the likelihood of short interval pregnancy. 3,4

\section{Methods:}

Setting: The study was conducted in coordination with a maternal health program, referred to as Madres Sanas, which currently provides four antenatal and two postnatal visits to women living in the Southwest Trifinio region of Guatemala, in their homes. ${ }^{5}$

Participants: Those women aged $15-35$ who had received antepartum care through the program, delivered, and were being evaluated for their routinely scheduled forty-day postpartum visit were offered enrollment. ${ }^{1}$ This current analysis includes women who were not lost to follow-up and were able to be evaluated at 12 months post-enrollment.

Sample Size: 208 women were enrolled in the trial after they gave informed consent to participate in the study and to be followed for twelve months postpartum. ${ }^{2} 108$ women were enrolled in intervention clusters, and 100 women in the control clusters. ${ }^{2}$

Outcomes: Our pre-specified secondary outcome measures were overall contraceptive uptake (by type) among women at 12 months post-enrollment as well as contraceptive continuation, satisfaction, and pregnancy rates, by study arm. ${ }^{1}$ These outcomes pertain to the cluster and participant level. No change to these outcomes were made after the trial commenced.

Statistical Methods: We performed univariate comparisons with a mixed effects regression adjusted for cluster of overall contraceptive and implant use at 12 months, which converted the comparisons to risk 
ratios with $95 \%$ confidence intervals. We used descriptive statistics to produce counts and percentages of contraceptive uptake and use by intervention arm and by study timepoints, describing initial method choices and continuation rates in the intervention clusters as well as use of methods by three and 12 months in all study participants. These same methods were used to describe satisfaction with contraceptive choices and frequency of short interval pregnancy among study participants. STATA software version 15.2 (StataCorp LP, College Station, TX, USA) was used for analysis.

\section{Results:}

Figure 1 presents the participant flow through the trial. Of 208 women enrolled in the trial, 108 women received the intervention, and 12-month outcome data was available for 94 of them. In the control clusters, among 100 women who were enrolled, 9 were lost to follow-up by 12 months. The study populations have previously been described in terms of age, education, marital status, parity, obstetric history, prenatal care receipt, and pregnancy outcomes. ${ }^{2}$

Table 1 presents the 12-month outcome of the study. Overall contraceptive use by 12 months was $59.0 \%$ in control clusters and $67.6 \%$ in intervention clusters (RR 1.1 [1.0,1.3], $p<0.05$ ). The table then shows how that usage breaks down by method of contraception including modern and traditional methods, which differed primarily with respect to implant usage (RR $1.5[1.2,2.0], p=0.002)$. The use of short-acting methods did not vary by study arm with respect to use by 12 months (RR 1.1 [0.9,1.2], p = 0.6). Implant usage by 12 months was statistically significantly different between study arms with 3 women from control clusters using the method compared to 25 women in the intervention arm (RR 1.6 [1.3,1.9], $p<$ 0.001). Of note, no adverse outcomes were reported; pregnancy occurred among 4 women in the control group and no women in the intervention group (RR $1.0[1.0,1.1], p=0.07)$.

Table 3 presents satisfaction outcomes collected at 12 months. There was no difference in satisfaction of women with contraceptive use between arms with about $95 \%$ of women very satisfied or a little satisfied in each arm. Those who were dissatisfied report being so due to side effects of nausea, amenorrhea, or heavy bleeding.

Table 4 illustrates why women who were not using a contraceptive method at 12 months declined to do so. There was no statistical difference in reasoning between the groups, with the most common reasons being that the women reportedly did not have a partner and therefore did not require contraception (26.4\%) and a non-specified reason of 'other' (37.7\%). Less common reasons included 'desiring pregnancy' or 'partner not supporting contraceptive use' (each 3.8\%) and 'being afraid of side effects' or 'not knowing why they chose not to use a method' (each 9.4\%).

Tables 5 and 6 present continuation rates of contraceptive methods that women in the intervention clusters chose at their enrollment visit. Of 2 women who initially chose condoms, neither of them was still using that method at 12 months (Table 5) and 1 had switched the injection (Table 6). Of 5 women who chose to initiate pills, 4 were still using the method at 12 months (Table 5). For the 37 women who 
received an injection at their enrollment visit, $57 \%$ were still using that method by 12 months (Table 5 ), and 2 had undergone sterilization by that time (Table 6). Of women who chose the implant at enrollment (30), 25 were still using that method by 12 months, with one woman discontinuing in favor of sterilization (Table 6).

\section{Discussion:}

Our cluster-randomized parallel-arm pragmatic trial designed to test the hypothesis that reducing barriers to accessing the contraceptive implant would increase usage at 3 months was shown to be a positive trial. ${ }^{2}$ By 12 months, the intervention clusters had a borderline higher overall contraceptive uptake rate, with significantly higher rates of implant use in the intervention clusters, but no difference in rates of short-acting contraceptive utilization between arms. In intervention clusters, contraceptive continuation rates ranged from $57.0 \%-83.0 \%$ with the highest continuation among implant users, and over $90 \%$ of all participants were very or a little satisfied across the entire study population. There was a trend toward a significant association with a reduced frequency of short interval pregnancy in the intervention compared to control clusters.

While we found borderline significance in overall contraceptive uptake at 12 months between the arms, we feel this likely due to the fact that the Madres Sanas program has been in effect since 2011 and postpartum contraceptive use is historically high this population, with many women also meeting World Health Organization recommendations for proper pregnancy spacing. ${ }^{2,6}$ Our results suggest that the intervention was still having a positive impact 12 months after initiation. It is possible that our home nursing prenatal program been successful at increasing contraceptive use in the region overall over the past decade. ${ }^{2,7}$

As explored in our primary outcome paper, the Pan American Health Organization has previously suggested that a focus on long-acting reversible contraceptives in Latin America is a priority. ${ }^{8}$ We felt that our current study and prior research responded to this call and provided evidence that home- and community-based nursing programs are an implementation strategy that can achieve high satisfaction and continuation rates of postpartum contraception. ${ }^{3,4}$ Next steps for research would be to focus on adaptation of our program to other settings and even other interventions such as adolescent pregnancy prevention, to determine in similar results are replicable.

While we report a good follow-up rate in the study, we do feel our results are potentially limited by missing data and relatively small sample sizes. For example, our short interval pregnancy was borderline reduced by our intervention, and it is possible that if we had more complete data, we would be able to assert that our intervention had reduced this outcome. It would also be good to have collected more comprehensive data on different methods of contraceptive use between three and 12 months to get a sense of contraceptive behavior around switching methods, though we have some sense that women in these communities often switched in favor of a more long-acting or permanent method than that which they originally initiated. 
In conclusion, our study, originally designed to respond to previously identified barriers to implant uptake, was successful at increasing the use of this long-acting method through 12 months postpartum. Not only did it increase overall use of contraception by three months, but it almost continued to do so at 12 months, still shifting contraceptive use away from shorting-acting methods in favor of longer-acting methods, with high continuation and satisfaction rates and no adverse outcomes reported. There was a trend toward a significant association with reduced frequency of short interval pregnancy. We believe that our findings support taking this approach to scale, especially in regions that are experiencing high rates of short interval pregnancy and low long-acting contraceptive utilization in Guatemala and beyond.

\section{Abbreviations:}

Not Applicable

\section{Declarations:}

\section{Ethics:}

The study protocol, the data collection forms, and the consent form were approved by both the Colorado Multiple Institutional Review Board and their International Research Advisory Committee (COMIRB \# 17 1314) in the United States of American as well as by the Instituto de Nurtición de Centro América y Panamá (INCAP) in Guatemala (CIE-REV 076/2018), and by the Community Advisory Board of the Madres Sanas program in the Southwest Trifinio. Both ethics review committees are providing ongoing review of the study as it is being conducted

\section{Consent for Publication:}

Not Applicable

\section{Data Availability:}

Data available on request due to privacy/ethical restrictions

\section{Competing Interests:}

The authors have no relationships to disclose that may be deemed to influence the objectivity of this paper and its review. The authors report no commercial associations, either directly or through immediate family, in areas such as expert testimony, consulting, honoraria, stock holdings, equity interest, ownership, patent-licensing situations or employment that might pose a conflict of interest to this analysis.

Additionally, the authors have no conflicts such as personal relationships or academic competition to disclose. The findings presented in this paper represent the views of the named authors only, and not the views of their institutions or organizations. A consent for publication section was not necessary. No individual-level data is presented in this manuscript, only de-identified aggregated data of women who consented to participate in the study and publication of the analysis. 


\section{Funding:}

Funding for this project comes from the Eunice Kennedy Shriver National Institutes of Child Health and Human Development Women's Reproductive Health Research K12 award (5K12HD001271) and the Doris Duke Charitable Foundation.

\section{Author Contributions:}

MSH and JS conceived of the project with feedback from SBM, CR, EA, GH, and SB. SBM and CR coordinate the study with oversight from $\mathrm{MSH}$ and $\mathrm{AB}$, and data management assistance from $\mathrm{AJZ}$. GH aided with the site initiation visit and interpretation of results. MSH performed the analysis and wrote the manuscript with input from all authors.

\section{Acknowledgements:}

We want to thank all the women and men involved in collection of the data analyzed in this work and all the women who participated in the study-their health, well-being, and successful pregnancy outcomes are the motivation for performing this work.

\section{References:}

1. Harrison MS, Bunge-Montes S, Rivera C, et al. Delivery of home-based postpartum contraception in rural Guatemalan women: a cluster-randomized trial protocol. Trials. 2019;20(1):639.

2. Harrison MS, Bunge-Montes S, Rivera C, et al. Primary and secondary three-month outcomes of a cluster-randomized trial of home-based postpartum contraceptive delivery in southwest Trifinio, Guatemala. Reprod Health. 2020;17(1):127.

3. Vernon R. Meeting the family planning needs of postpartum women. Studies in family planning. 2009;40(3):235-245.

4. Peipert JF, Zhao Q, Allsworth JE, et al. Continuation and satisfaction of reversible contraception. Obstet Gynecol. 2011;117(5):1105-1113.

5. Asturias EJ, Heinrichs G, Domek G, et al. The Center for Human Development in Guatemala. Advances in Pediatrics. 2016;63(1):357-387.

6. Harrison MS, Montes SB, Rivera C, et al. Interpregnancy Interval in a Rural Guatemalan Population: Results from a Quality Improvement Database. Matern Child Health J. 2020;24(8):1038-1046.

7. Schultz C, Larrea N, Celada M, Heinrichs G. A Qualitative Assessment of Community Attitudes and Barriers to Family Planning Use in the Trifinio Region of Southwest Guatemala. Maternal and Child Health Journal. 2018;22(4):461-466.

8. Ponce De Leon RG, Ewerling F, Serruya SJ, et al. Contraceptive use in Latin America and the Caribbean with a focus on long-acting reversible contraceptives: prevalence and inequalities in 23 countries. The Lancet Global Health. 2019;7(2):e227-e235. 


\section{Tables:}


Table 1.

Contraceptive Use and Repeat Pregnancy by Twelve Months Post-Enrollment

\begin{tabular}{|c|c|c|c|c|c|}
\hline & $\begin{array}{l}\text { Total Population of } \\
\text { Women Followed to } \\
12 \text { Months } \\
(n=185)\end{array}$ & $\begin{array}{l}\text { Control Clusters (4) } \\
\text { Women Followed to } \\
12 \text { Months } \\
(\mathrm{n}=91)\end{array}$ & $\begin{array}{l}\text { Intervention } \\
\text { Clusters (4) } \\
\text { Women } \\
\text { Followed to } \\
12 \text { Months } \\
(n=94)\end{array}$ & $\begin{array}{l}\text { P- } \\
\text { Value }\end{array}$ & $\begin{array}{l}\mathrm{RR} \\
{[95 \%} \\
\mathrm{Cl}]\end{array}$ \\
\hline $\begin{array}{l}\text { Using a } \\
\text { Method by } 12 \\
\text { Months } \\
\text { Yes (\% of } \\
\text { total) }\end{array}$ & 132 (63.5\%) & 59 (59.0\%) & 73 (67.6\%) & 0.05 & $\begin{array}{l}1.1 \\
{[1.0} \\
1.3]\end{array}$ \\
\hline $\begin{array}{l}\text { Method Being } \\
\text { Used at } 12 \\
\text { Months }\end{array}$ & $1(0.8 \%)$ & $1(0.8 \%)$ & $0(0.0 \%)$ & 0.002 & $\begin{array}{l}1.5 \\
{[1.2,2.0]}\end{array}$ \\
\hline Abstinence & 4 (3.1\%) & $0(0.0 \%)$ & $4(5.6 \%)$ & & \\
\hline Pills & $90(70.3 \%)$ & $52(91.2 \%)$ & 38 (53.5\%) & & \\
\hline Injection & $28(21.9 \%)$ & 3 (5.3\%) & 25 (35.2\%) & & \\
\hline Implant & $1(0.8 \%)$ & $0(0.0 \%)$ & $1(1.4 \%)$ & & \\
\hline Intrauterine & $4(3.1 \%)$ & $1(1.8 \%)$ & 3 (4.2\%) & & \\
\hline $\begin{array}{l}\text { Female } \\
\text { Sterilization }\end{array}$ & $4(3.1 \%)$ & 2 (3.6\%) & $2(2.8)$ & & \\
\hline Missing & & & & & \\
\hline $\begin{array}{l}\text { Short-Acting } \\
\text { Method at } 12 \\
\text { Months* }\end{array}$ & 94 (71.2\%) & $52(88.1 \%)$ & 42 (57.5\%) & 0.6 & $\begin{array}{l}1.1 \\
{[0.9,1.2]}\end{array}$ \\
\hline $\begin{array}{l}\text { Yes (\% of } \\
\text { users) }\end{array}$ & & & & & \\
\hline $\begin{array}{l}\text { Implant at } 12 \\
\text { Months } \\
\text { Yes (\% of } \\
\text { users) }\end{array}$ & 28 (\%) & $3(\%)$ & $25(\%)$ & $<0.001$ & $\begin{array}{l}1.6 \\
{[1.3,1.9]}\end{array}$ \\
\hline $\begin{array}{l}\text { Pregnant by } \\
12 \text { months } \\
\text { Yes (\% of } \\
\text { total) }\end{array}$ & $4(2.2 \%)$ & 4 (4.4\%) & $0(0.0 \%)$ & 0.07 & $\begin{array}{l}1.0 \\
{[1.0,1.1]}\end{array}$ \\
\hline
\end{tabular}


Note: $p$-values the result of a generalized linear model mixed effects regression adjusted for cluster

*: Short Acting Method Includes: condoms, pills, injection

Table 3.

Satisfaction of women on contraception by twelve months post-enrollment

\begin{tabular}{|c|c|c|c|c|c|}
\hline & $\begin{array}{l}\text { Women on } \\
\text { Contraception at } \\
\text { Twelve Months } \\
(n=132)\end{array}$ & $\begin{array}{l}\text { Control Clusters (4) } \\
\text { Women on } \\
\text { Contraception at } \\
\text { Twelve Months } \\
(n=59)\end{array}$ & $\begin{array}{l}\text { Intervention } \\
\text { Clusters (4) } \\
\text { Women on } \\
\text { Contraception at } \\
\text { Twelve Months } \\
(n=73)\end{array}$ & $\begin{array}{l}\text { P- } \\
\text { Value }\end{array}$ & $\begin{array}{l}\mathrm{RR} \\
{[95 \%} \\
\mathrm{Cl}]\end{array}$ \\
\hline Satisfaction & & & & 1.0 & 1.0 \\
\hline (c) & 103 (78.0\%) & 45 (76.3\%) & 58 (79.5\%) & & {$[0.8,1.2]$} \\
\hline Satisfied & $22(16.7 \%)$ & $12(20.3 \%)$ & $10(13.7 \%)$ & & \\
\hline A Little & $5(3.8 \%)$ & $1(1.7 \%)$ & $4(5.5 \%)$ & & \\
\hline $\begin{array}{l}\text { A Little } \\
\text { Dissatisfied }\end{array}$ & $2(1.5 \%)$ & $1(1.7 \%)$ & $1(1.4 \%)$ & & \\
\hline $\begin{array}{l}\text { Very } \\
\text { Dissatisfied }\end{array}$ & & & & & \\
\hline $\begin{array}{l}\text { If } \\
\text { Dissatisfied, } \\
\text { Why? }\end{array}$ & 1 (14.3\%) & $1(50.0 \%)$ & 0 (0.0\%) & - & - \\
\hline Nausea & 2 (28.6\%) & $1(50.0 \%)$ & $1(20.0 \%)$ & & \\
\hline $\begin{array}{l}\text { Amenorrhea } \\
\text { Heavy } \\
\text { Bleeding }\end{array}$ & $4(57.1 \%)$ & $0(0.0 \%)$ & $4(80.0 \%)$ & & \\
\hline
\end{tabular}

Note: p-values the result of a generalized linear model mixed effects regression adjusted for cluster 
Table 4.

Reasons women not using contraception were not doing so by twelve months post-enrollment

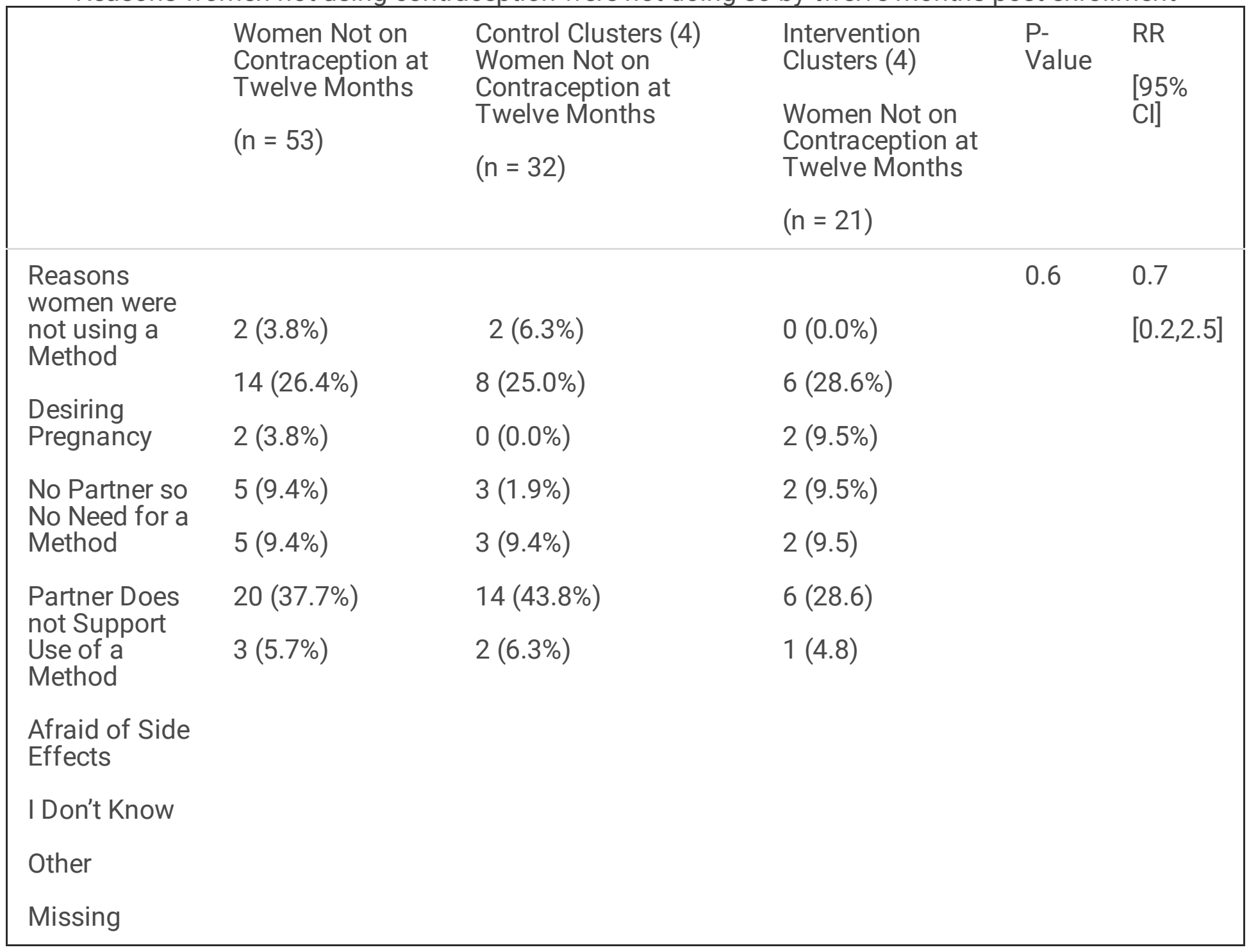

Note: p-values the result of a generalized linear model mixed effects regression adjusted for cluster 
Table 5.

Continuation rates of methods chosen by women at their enrollment visit in intervention clusters

Number of women who started a method at their enrollment visit who were still on that method at 12 months post-enrollment

Contraceptive Method

Chosen at

Enrollment Visit
Three Month

Continuation Rate of Enrollment Method

Condoms 2

Pills

Injection

Implant

2

Condoms

$1,50 \%$

Pills

$4,80 \%$

Injection

$28,76 \%$

$27,90 \%$

Implant

30

Table 6.

Initial contraceptive choice of women in intervention clusters and method they switched to by twelve months post-enrollment

\begin{tabular}{|c|c|c|c|c|c|}
\hline & \multicolumn{5}{|c|}{$\begin{array}{l}\text { Contraceptive Choice } 12 \text { Months Post-Enrollment (Switched to this Method by } 12 \\
\text { Months) }{ }^{a}\end{array}$} \\
\hline & Pills & Injection & Implant & IUD & Tubal Ligation \\
\hline \multicolumn{6}{|c|}{ Initial Contraceptive Choice at Enrollment } \\
\hline $\begin{array}{l}\text { No } \\
\text { Method }\end{array}$ & 0 & 17 & 0 & 1 & 0 \\
\hline Condoms & 0 & 1 & 0 & 0 & 0 \\
\hline Pills & 4 & 0 & 0 & 0 & 0 \\
\hline Injection & 0 & 21 & 0 & 0 & 2 \\
\hline Implant & 0 & 0 & 25 & 0 & 1 \\
\hline
\end{tabular}

a. choices of two women missing

Table 2 not available with this version.

\section{Figures}




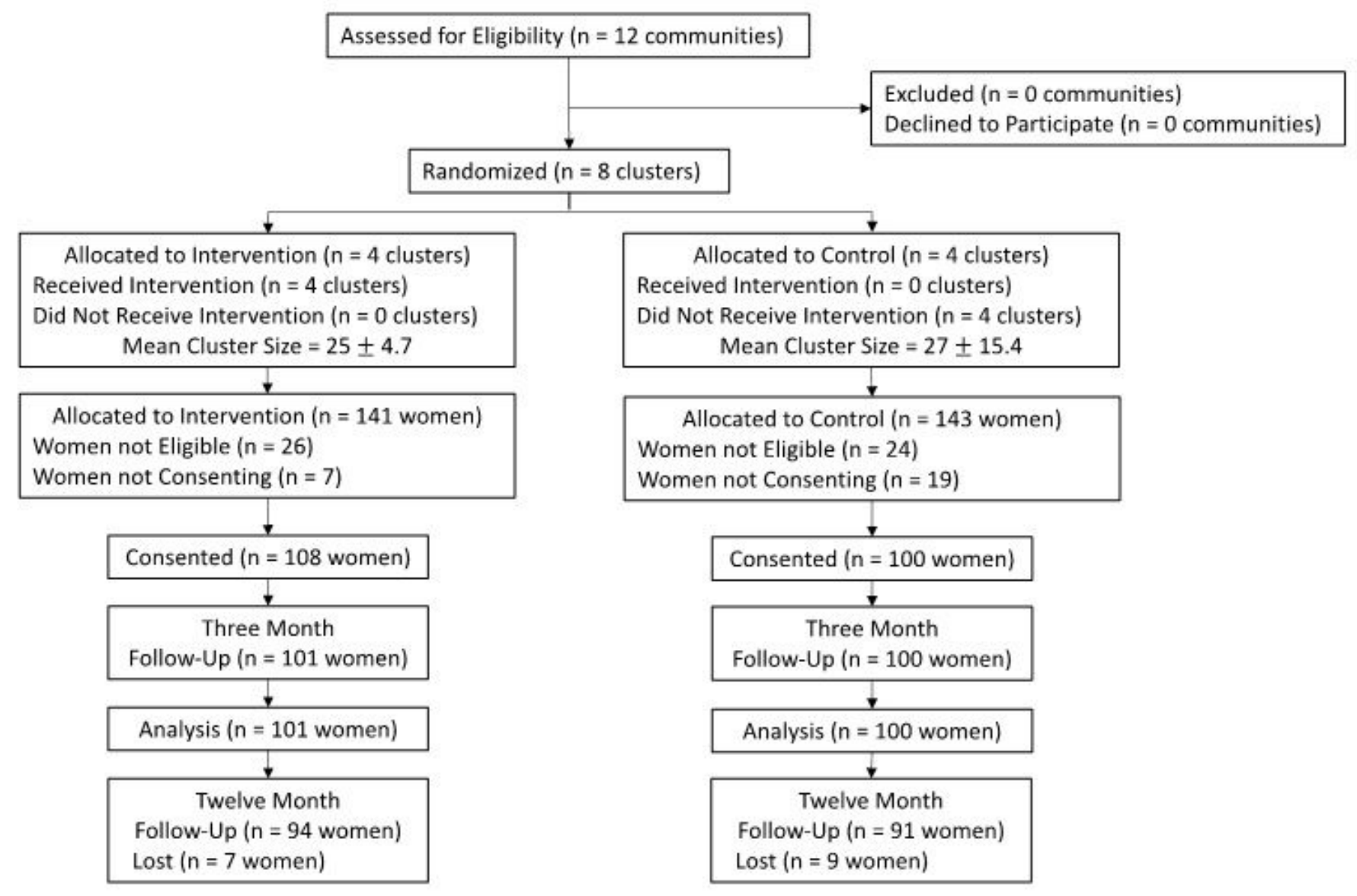

Figure 1

CONSORT DIAGRAM

\section{Supplementary Files}

This is a list of supplementary files associated with this preprint. Click to download.

- CONSORTExtensionforClusterTrials2012ChecklistRH.docx 